\title{
A guide to diagnosing clinical parasites
}

\author{
Reviewed by Jon D. Reuter, DVM, MPVM, DACLAM
}

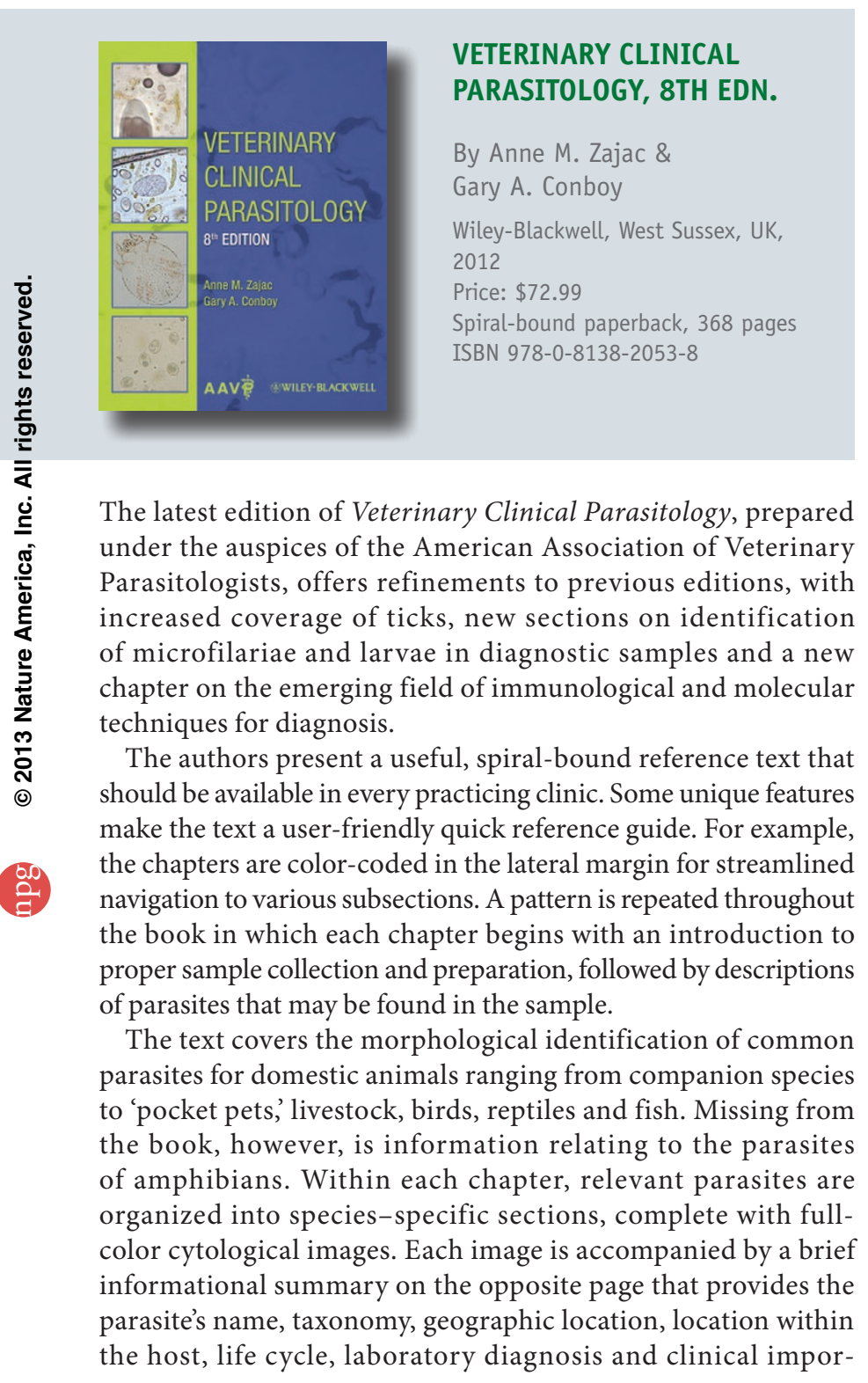

tance. In addition to the photographs, very fine, detailed line drawings that highlight specific, unique anatomical structures intermittently augment the chapters. The details regarding life cycle are very limited, with little to no coverage of developmental stages or intermediate hosts.

The first and third chapters group parasites by host species. This pattern is very helpful for quickly locating specific organisms. In the remaining chapters, the structure deviates from this pattern; one must read the figure legends to find species-specific information about each parasite presented in these chapters. Arthropods and fish each are given their own separate chapters. Although they diverge from the standard layout, these chapters are very useful because they present animal-specific techniques and parasites in one area for quick and easy reference. This book would benefit from reorganizing all of the chapters to separate parasites by host species.

The new chapter on immunological and molecular diagnostic techniques is very brief, with a simple overview of enzyme-linked immunosorbent assays and antigen- and antibody-related assays. It omits many of the advanced diagnostic methods and only briefly mentions polymerase chain reaction, one of the fastest growing and most sensitive diagnostic testing approaches. A table is included in an attempt to provide a summary of the diagnostic tests available in the US, but this table is outdated. Furthermore, it has virtually no information about laboratory animals or guidance to readers about diagnostic labs that offer these services.

As a clinical reference text, the book should include some additional discussion of therapeutic approaches. Instead, readers will need to consult other reference texts or visit the Companion Animal Parasite Council website (http://www.capcvet.org/) which also provides a discussion of potential health concerns related to zoonosis.

Though great for assisting veterinarians and technicians with the diagnosis of the most common clinical parasites, the book provides only a cursory overview for the common laboratory animal species, with its major focus on companion animals. For an in-depth presentation of laboratory animal species, readers should consult standard reference texts such as Flynn's Parasites of Laboratory Animals, $2^{\text {nd }}$ ed. (ed. Baker, D.; 2007). 